\title{
High prevalence of multi-drug resistant bacteria in selected poultry farms in Selangor, Malaysia.
}

\begin{abstract}
Prevalence of multidrug resistant bacteria in apparently healthy chickens from 3 selected poultry farms in Selangor area of Malaysia was investigated. Conventional isolation techniques such as growth on selective media, gram staining and biochemical tests was utilised for the identification of the different bacterial isolates. Antimicrobial sensitivity test was monitored with the disc diffusion assay against 12 antimicrobial agents. A total of 96 Staphylococcus aureus, 48 E.coli, 7 Pasteurella sp. and 6 Salmonella sp. were isolated. All E. coli and Salmonella spp. isolates were multidrug resistant while $77.2 \%$ of Staphylococcus aureus and $71.5 \%$ of Pasteurella sp. isolates were multidrug resistant. The study further reveled highest resistance to tetracycline while cephatothin as the best drug of choice for treatment of infections caused by the isolates in the study area. Since not only chickens are at risk, this study recommends urgent intervention by regulatory agencies to limit the emergence and spread of these bacteria as well as prudent use of antibacterial agents among farmers in Malaysia.
\end{abstract}

Keyword: Multidrug; Resistant; Bacteria; Chickens; Malaysia. 